\title{
Management of Project Schedule Exceptions based on Bayesian Networks
}

\begin{abstract}
[ Chulsoon Park, Hongsuk Sung, Sungmoon Bae, Sangcheon Lee ]
Abstract - The market size of plant projects in overseas is so large that domestic EPC project contractors are actively seeking the overseas projects and then trying to meet completion plans since successful fulfillment of these projects can provide great

Hours per day batch production system developed unattended continuous production of large aircraft parts) funded by the Ministry of Trade, Industry and Energy in 2016-2019, and also by Changwon National University in 2017.
\end{abstract} opportunities for them to expand into new foreign markets. International EPC projects involve all of the uncertainties common to domestic projects as well as uncertainties specific to foreign projects including marine transportation, customs, regulations, nationality, culture and so on. When overseas project gets off-schedule, the resulting uncertainty may trigger unexpected exceptions and then critical effects to the project performance. It usually require much more time and costs to encounter these exceptions in foreign sites compared to domestic project sites. Therefore, an exception handling approach is required to manage exceptions effectively for successful project progress in foreign project sites.

In this research, we proposed a methodology for prediction and evaluation of exceptions caused by risks in international EPC projects based on sensitivity analysis and Bayesian Networks. First, we identified project schedule risks and related exceptions, which may meet during the fulfillment of foreign EPC projects that is performed in a sequence of engineering, procurement, preparatory manufacture, foreign shipping, construction, inspection and modification activities, and affect project performance, using literature review and expert interviews. The impact of exceptions to the schedule delay were also identified. Second, we proposed a methodology to predict the occurrence of exceptions caused by project risks and evaluate them. Using sensitivity analysis, we can identify activities that critically affect schedule delay and need to focus by priority. Then, we use Bayesian Networks to predict and evaluate exceptions. Third, we applied the proposed methodology to an international EPC project example to validate the proposed approach. Finally, we concluded the research with the further research topics. We expect that the proposed approach can be extended to apply in exception management in project management.

Keywords-Bayesian Networks, Exception Handling, Sensitivity Analysis, Schedule Prediction

\section{Acknowledgment}

This work was supported by the Industrial Strategic Technology Development Program (10067408, Available 24
About Author :

Prof. Chulsoon Park is currently with School of Industrial Engineering and Naval Architecture, Changwon National University, South Korea

His main research topics include Condition based Monitoring, Predictive Maintenance, Bayesian Networks.

Dr. Hongsuk Sung was graduated from School of Industrial Engineering and Naval Architecture, Changwon National University

His main reseach topics include Project Management, Bayesian Networks

Prof. Sungmoon Bae is currently with School of Industrial and Systems Engineering, Gyeongsang National University, South Korea

His main research topics include Object Oriented Technology, System Analsys and Design, System Integration.

Prof. Sangcheon Lee is currently with School of Industrial and Systems Engineering, Gyeongsang National University, South Korea

His main research topics include Reliability Engineering, Quality Management, Industrial Statistics 